\title{
Tunable Reflection Type Plasmon Induced Transparency with Graphene
}

\author{
$\underline{\text { M. Habib }}^{1,2}$, E. Ozbay $^{1,2,3}$ H. Caglayan ${ }^{4}$ \\ ${ }^{1}$ Department of Electrical and Electronics Engineering, Bilkent University, Ankara 06800, Turkey \\ ${ }^{2}$ Nanotechnology Research Center, Bilkent University, Ankara 06800, Turkey \\ ${ }^{3}$ Department of Physics, Bilkent University, Ankara 06800, Turkey \\ ${ }^{4}$ Laboratory of Photonics, Tampere University of Technology, 33720, Tampere, Finland \\ mohsin.habib@bilkent.edu.tr
}

\begin{abstract}
Plasmon induced transparency (PIT) has always been investigated in transmission mode, which makes the design dependent upon the type of substrate. In this work, we propose a first tunable reflection type PIT (RPIT) device based on simple design of two parallel gold strips on graphene. We have numerically investigated the design by using Finite Difference TimeDomain (FDTD) method. This response is tunable by changing the Fermi level $\left(E_{f}\right)$ of graphene. High tunability of $215 \mathrm{~nm}$ shift was observed by changing the $E_{f}$ of graphene from 0 to $0.8 \mathrm{eV}$. The spectral contrast ratio of our device is $\mathbf{9 9 . 2} \%$.
\end{abstract}

\section{INTRODUCTION}

Electromagnetic induced transparency (EIT) is a quantum destructive interference phenomena taking place in a medium excited by two lasers having different transitions [1]. Plasmon induced transparency (PIT) is similar effect as EIT which has been proposed in variety of plasmonic structures. Recently, PIT has been investigated by several groups based on metallic nanoparticles [2,3] and metamaterial structures [4,5]. The design based on metamaterials structure are realized by destructive interference of dark-bight modes and detuning of two bright modes to achieve PIT response. The resonances of these modes can be manipulated by design and dimensions of structures. Moreover, dynamic tunability of PIT has been proposed based on hybrid metal-graphene designs [5]. Dynamically tunable PIT devices can be used for tunable sensing and switchable systems.

Recently tunable PIT devices based on graphene have been numerically investigated. Most of these designs are working in transmission mode [7, 8]. Reflection type PIT (RPIT) has been realized only by Ding et al. in 2014 [9]. In this design, cut wire and split ring resonators have been used as dark and bright modes respectively. RPIT response of the device by destructive interference of the modes have been observed theoretically. However, the design was not dynamically tunable. Therefore, in this work we propose a hybrid metal-graphene based design to generate tunable RPIT response for the first time. The RPIT response of device is in MIR range that is tunable by changing $E_{f}$ of graphene.

Graphene is a 2-D material that has been regarded as new class of promising plasmonic material. Graphene has tight field confinement and a controllable Fermi level $\left(E_{f}\right)$ with gate voltage at mid infrared (MIR) regime. Graphene $E_{f}$ is a direct function of charge density as shown in, Equation 1:

$$
E_{f}=\hbar \nu_{F} \sqrt{\pi n}
$$

where $\hbar$ is reduced Planck's constant, $n$ is charge density and $\nu_{\mathrm{F}}$ is Fermi velocity that is $-1.1 \times 10^{8} \mathrm{~cm} \mathrm{~s}^{-1}$ [6]. Therefore, graphene is suitable material for dynamic tunable RPIT.

\section{Design And Numerical Simulations}

We have numerically investigated RPIT design using Finite Difference Time-Domain (FDTD) method. The unit cell of the design has two parallel strips of gold (Au) serving as two bright, shown in Fig. 1(a). The lengths of strips are selected as 0.8 and $1 \mu \mathrm{m}$, width as $\mathrm{W}=200 \mathrm{~nm}$ for both the strips and distance $\mathrm{D}=200 \mathrm{~nm}$ between the two strips. The unit cell has periodic boundary condition in $x$ and $y$-axes and Perfect Matched Layers (PML) in the 

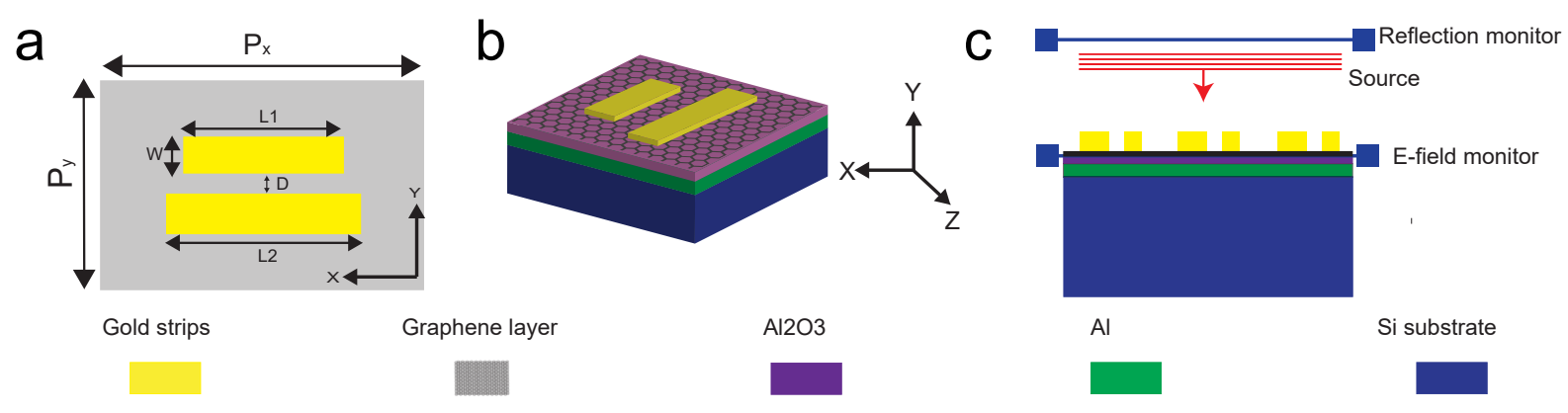

Fig. 1: (a) Unit cell of PIT structure. Two Au strips (golden) with lengths of L1 $(0.8 \mu \mathrm{m}), \mathrm{L} 2(1 \mu \mathrm{m}), \mathrm{W}(200 \mathrm{~nm})$ is width and D $(200 \mathrm{~nm})$ is distance between two strips. Px and Py show periodicity of unit cell. (b) 3D view of RPIT device and (c) simulation setup of the design.

direction of propagation (z-axis). The design has periodicity of $1.5 \mu \mathrm{m}$ in $x$-axis and $1.0 \mu \mathrm{m}$ in $y$-axis. The unit structure of device has four layers on top of Si substrate. From top to bottom, this structure has $70 \mathrm{~nm}$ thick $\mathrm{Au}$ strips, graphene layer as 2-D dispersive material, $50 \mathrm{~nm}$ of aluminum oxide $\left(\mathrm{Al}_{2} \mathrm{O}_{3}\right)$ layer and 100 of reflecting $\mathrm{Al}$ layer, shown in Fig. 1 (b). A plane wave source along the $z$ direction was used to illuminate the unit cell having the electric field component $(E)$ parallel to $x$-axis. Scattering rate of graphene was selected as $0.01 \mathrm{eV}$ and Fermi level was changed from $0 \mathrm{eV}$ to $0.8 \mathrm{eV}$. The simulation setup is shown in Fig. 1(c).

\section{RESULTS}

The reflection simulation of the proposed design is presented in Fig. 2. Both the strips have own resonances that can be controlled by the length and width of strips presented in the inset of Fig. 2. When we bring two strips close to each other, they result in weak hybridization and produce PIT in reflection mode. This RPIT response is not present when we excite the single strips individually. We have carefully designed the lengths, width and distance between the strips to achieve in RPIT response in MIR range so that we can tune by changing the $E_{f}$ of graphene. A blue shift was observed when $E_{f}$ was increased from 0 to $0.8 \mathrm{eV}$. Total shift of $215 \mathrm{~nm}$ was observed.

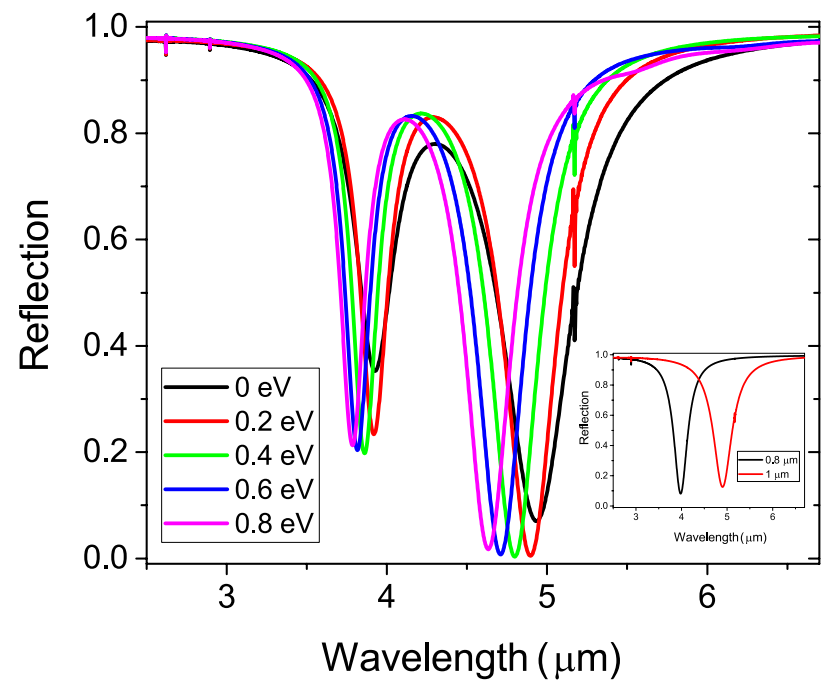

Fig. 2: Reflection of single strips $0.8 \mu \mathrm{m}$ in black and $1 \mu \mathrm{m}$ in red (inset). Simulated RPIT results at different $E_{f}$ of graphene.

E-field investigation of two strips were made for three different wavelengths, shown in Fig. 3. Electric field magnitudes were presented at of two resonance wavelengths (3.86 $\mu \mathrm{m}$ and $4.79 \mu \mathrm{m})$ in Fig. 3(a) and 3(c). Moreover, 
the E-field magnitude is shown at RPIT wavelength $(4.29 \mu \mathrm{m})$ in Fig. 3(b).

a

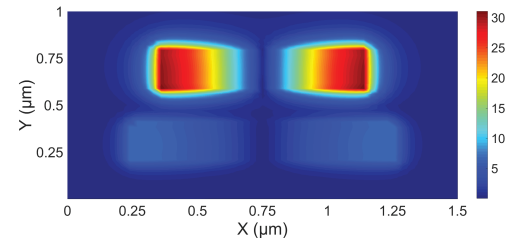

b

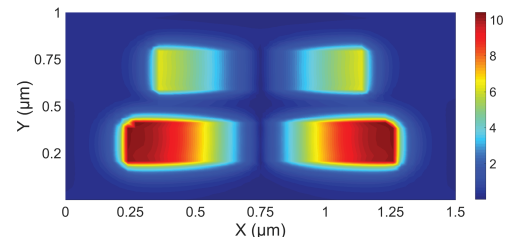

C

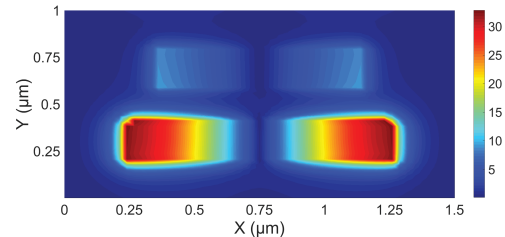

Fig. 3: E-field magnitude at $0.4 \mathrm{eV}$ (a) E-field magnitude at $3.86 \mu \mathrm{m}$. (b) E-field magnitude at $4.29 \mu \mathrm{m}$ (c) E-field magnitude at $4.79 \mu \mathrm{m}$.

In order to model, our design we follow the concept from paper [9], in which they have treated similar type of structures as Fabry-Perot resonator. There are two surfaces that reflect the electromagnetic waves, one of them is partially reflecting (Au gold strips) and completely reflecting surface (Al layer at the bottom). The patterned structure will reflect partial wave and rest of the waves will go inside the resonator and will appear after multiple internal reflection. Therefore, the total response of the structure is superposition of partial reflection from surface and multiple reflections inside the structure before they come out in air. Which means our design is based on multi-reflection theory for understanding physical mechanism of reflection type PIT in a better way.

The performance of RPIT device can be evaluated by spectral contrast ratio $\left(S_{c o n}\right)$ for optical application, SCR is described as:

$$
S_{\text {con }}=\frac{\left(R_{\text {peak }}-R_{\text {dip }}\right)}{\left(R_{\text {peak }}+R_{\text {dip }}\right)} \times 100
$$

where $R_{\text {peak }}$ is intensity of reflection peak and $R_{\text {dip }}$ is intensity of resonance dip. $S_{\text {con }}$ of our design is $99.2 \%$. The device with high $S_{c o n}$ is suitable for filtering and switching applications.

\section{CONCLUSION}

In this work we have numerically investigated PIT in reflection mode (RPIT) by simple two strips on top of graphene. By changing $E_{f}$ of graphene PIT in reflection mode was response was shifted. The results of tunable RPIT can be used in many fields such as slow light applications and nonlinear optics.

\section{ACKNOWLEDGEMENT}

This work is supported by "COMPETITIVE FUNDING TO STRENGTHEN UNIVERSITY RESEARCH PROFILES funded by Academy of Finland, decision number 301820.”

\section{REFERENCES}

[1] K. Boller, a Imamolu, and S. Harris, "Observation of electromagnetically induced transparency," Phys. Rev. Lett., vol. 66, no. 20, pp. 2593-2596, 1991.

[2] V. Yannopapas, E. Paspalakis, and N. V. Vitanov, "Electromagnetically induced transparency and slow light in an array of metallic nanoparticles," Phys. Rev. B, vol. 80, no. 3, pp. 35104, 2009.

[3] C. Rohde, K. Hasegawa, and M. Deutsch, "Plasmon-assisted transparency in metal-dielectric microspheres" Opt. Lett., vol. 32, no. 4, pp. 415-417, 2007.

[4] J. Xie, X. Zhu, X. Zang, Q. Cheng, Y. Ye, and Y. Zhu, "High extinction ratio electromagnetically induced transparency analogue based on the radiation suppression of dark modes," Sci. Rep., vol. 7, no. 1, pp. 1-8, 2017.

[5] M. Habib, A.R. Rashed, E. Ozbay, and H. Caglayan, "Graphene-based tunable plasmon induced transparency in gold strips. ," Optical Materials Express, vol. 8, no 4, pp.1069-1074, 2018.

[6] V. E. Dorgan, M. H. Bae, and E. Pop, "Mobility and saturation velocity in graphene on SiO2," Appl. Phys. Lett., vol. 97, no. 8 , pp. 811, 2010.

[7] X. Shi et al., Plasmonic analog of electromagnetically induced transparency in nanostructure graphene, Opt. Express, vol. 21, no. 23, pp. 2843828443, 2013.

[8] M. Id et al., Graphene-based tunable terahertz plasmon-induced transparency metamaterial, pp. 1527315280, 2016.

[9] C. F. Ding, Y. T. Zhang, J. Q. Yao, C. L. Sun, D. G. Xu, and G. Z. Zhang, Reflection-type electromagnetically induced transparency analogue in terahertz metamaterials, Chinese Phys. B, vol. 23, no. 12, 2014. 